\title{
The Uniform geometrical Theory of Diffraction for elastodynamics: Plane wave scattering from a half-plane
}

\author{
Audrey Kamta Djakou a) and Michel Darmon \\ Department of Imaging and Simulation for Nondestructive Testing, CEA, LIST, 91191 Gif-sur-Yvette, France \\ Larissa Fradkin \\ Sound Mathematics Ltd., Cambridge CB4 2AS, United Kingdom \\ Catherine Potel ${ }^{\text {b) }}$ \\ Laboratoire d'Acoustique de l'Université du Maine (LAUM), UMR CNRS 6613, 72085 Le Mans cedex 9 , \\ France
}

\begin{abstract}
Diffraction phenomena studied in electromagnetism, acoustics, and elastodynamics are often modeled using integrals, such as the well-known Sommerfeld integral. The far field asymptotic evaluation of such integrals obtained using the method of steepest descent leads to the classical Geometrical Theory of Diffraction (GTD). It is well known that the method of steepest descent is inapplicable when the integrand's stationary phase point coalesces with its pole, explaining why GTD fails in zones where edge diffracted waves interfere with incident or reflected waves. To overcome this drawback, the Uniform geometrical Theory of Diffraction (UTD) has been developed previously in electromagnetism, based on a ray theory, which is particularly easy to implement. In this paper, UTD is developed for the canonical elastodynamic problem of the scattering of a plane wave by a half-plane. UTD is then compared to another uniform extension of GTD, the Uniform Asymptotic Theory (UAT) of diffraction, based on a more cumbersome ray theory. A good agreement between the two methods is obtained in the far field.
\end{abstract}

\section{INTRODUCTION}

The scattering of elastic waves from an obstacle is of great interest in ultrasonic non-destructive evaluation (NDE), the main scattering phenomena being specular reflection and diffraction. When both the wavefront of an incident wave and the boundary of the scattering object can be modeled as locally plane, the scattered field is usually simulated using the Kirchhoff Approximation (KA). This represents the field as an integral over the scattering surface. ${ }^{1}$ KA gives a reliable and continuous description of specular reflections and fictitious fields compensating the incident field in the obstacle shadow, which together form the socalled Geometrico-Elastodynamic (GE) field. However, the diffracted fields are not always well described. ${ }^{2,3}$ In the absence of interaction with other waves, the best description of diffracted fields is obtained via the Geometrical Theory of Diffraction (GTD). ${ }^{4}$ This postulates existence of rays diffracted from the structure irregularities such as edge or tip, additional to the incident and reflected rays and also gives a recipe for calculating the amplitudes carried by these rays. In elastodynamics the underlying canonical problem is the scattering of a plane wave by a stress-free half-plane. Its

\footnotetext{
a) Also at: Laboratoire d'Acoustique de l'Université du Maine (LAUM), UMR CNRS 6613, 72085 Le Mans cedex 9, France. Electronic mail: audrey.kamta-djakou@cea.fr

b) Also at: Fédération Acoustique du Nord Ouest (FANO), FR CNRS 3110, France.
}

solution is represented in the form of the Sommerfeld inte$\operatorname{gral}^{5,6}$ and the classical GTD solution is obtained using the steepest descent method. The resulting contribution of the stationary phase points represents the diffracted waves in the far field using the so-called diffraction coefficients, otherwise known as the directivity patterns.

It is well known that the method of steepest descent is inapplicable when the stationary phase point of the integrand coalesces with its pole, explaining why the classical GTD fails in the zones where edge diffracted waves interfere with incident or reflected waves. For this reason the classical GTD solution is said to be non-uniform. Several uniform extensions of GTD have been developed in electromagnetism, such as the Uniform Asymptotic Theory (UAT) ${ }^{7-9}$ based on the Van Der Waerden method, ${ }^{10}$ the Uniform Theory of Diffraction (UTD) $)^{11,12}$ based on the Pauli-Clemmow method ${ }^{13}$ and the Physical Theory of Diffraction (PTD), ${ }^{1,2,14}$ which combines GTD and Kirchhoff Approximation (KA) and applies to arbitrary large locally plane scatterers.

The elastodynamic versions of UAT (Ref. 6) and PTD (Ref. 3) have been reported before; in the leading order when the scatterer is a half-plane PTD is identical to UAT. ${ }^{3}$ Due to the integral nature of the method, PTD appears to be computationally expensive, especially when the scatterers are large. A procedure similar to PTD has been confusingly called by its author the Uniform Theory of Diffraction. ${ }^{15}$ It relies on another integral method, which is difficult to implement. Whenever applicable, ray methods are often preferred 


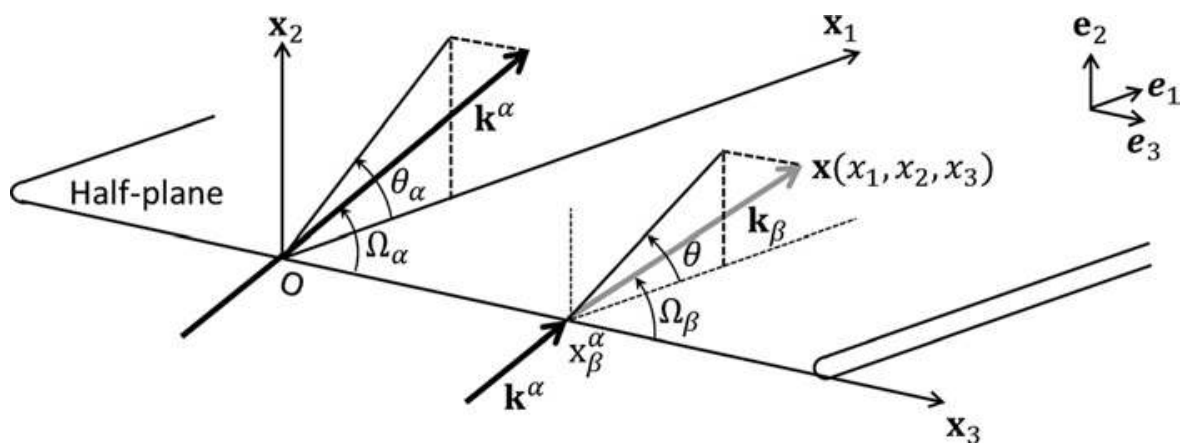

FIG. 1. A plane wave of propagation vector $\mathbf{k}^{\alpha}$ incident on a semi-infinite stress free crack. Thick black arrow, direction of the incident wave; thick gray arrow, direction of the diffracted wave $\left(\mathbf{k}_{\beta}\right)$.

to integral methods since highly optimized and therefore extremely fast ray tracing algorithms have become available. Both UAT and UTD are ray methods, producing continuous approximations to total fields. However, UAT involves artificial extension of the scattering surface and fictitious reflected rays (see Fig. 3 and the end of Sec. III), while UTD does not. For this reason, UTD is more used than UAT in acoustics ${ }^{16}$ and electromagnetism. ${ }^{17}$

The paper is structured as follows: in Sec. II, the exact solution of the canonical problem of a plane wave scattering from a stress-free half-plane is presented in the form of the Sommerfeld integral and its non-uniform asymptotics are given in the form convenient for use in the UTD recipe. In Sec. III, uniform asymptotics of the integral are derived using the Pauli-Clemmow procedure. As a result, the elastodynamic UTD is obtained, with the total field involving no fictitious rays and with diffraction coefficients, which are a simple modification of the GTD diffraction coefficients. Comparison of UTD and UAT is carried out in Sec. IV.

\section{SCATTERING OF A PLANE ELASTIC WAVE BY A HALF-PLANE CRACK: NON-UNIFORM ASYMPTOTICS}

In the following, the symbols $\alpha$ and $\beta$ are used to denote the wave type, i.e., $\alpha, \beta=\mathrm{L}$, TV, or TH (longitudinal, transverse vertical, or transverse horizontal, respectively). In general, $\alpha$ is used for the incident wave and $\beta$ for reflected and diffracted waves. Scalar quantities are generally labelled by taking $\alpha$ and $\beta$ as subscripts, while $\alpha$ is used as superscripts for vectors related to the incident wave and $\beta$ as subscripts for vectors related to scattered waves.

The geometry of the problem is presented in Fig. 1, using the Cartesian system based on an orthonormal basis $\left\{\mathbf{e}_{1}, \mathbf{e}_{2}, \mathbf{e}_{3}\right\}$ and the origin $\mathrm{O}$ at the crack edge. The crack is embedded in an elastic homogeneous and isotropic space and its face lies in the half-plane $\left\{x_{2}=0, x_{1} \geq 0\right\}$. The edge coincides with the $x_{3}$ axis and is irradiated by a plane wave

$$
\mathbf{u}^{\alpha}(\mathbf{x})=A \mathbf{d}^{\alpha} e^{i\left(-\omega t+\mathbf{k}^{\alpha} \cdot \mathbf{x}\right)}
$$

where $A$ is the wave displacement amplitude; $\mathbf{d}^{\alpha}$ is its polarization; $\mathbf{k}^{\alpha}$ is its wave vector defined thanks to the angles $\Omega_{\alpha}$ and $\theta_{\alpha}$ (described in Fig. 1), whose magnitude $k_{\alpha}=\omega / c_{\alpha}$, with $\omega$, the circular frequency and $c_{\alpha}$, the speed of the corresponding mode; $t$ is time; and $\mathbf{x}$ is the position vector, which is expressed in the Cartesian coordinates as $\left(x_{1}, x_{2}, x_{3}\right)$ and in the cylindrical coordinates as $\left(r, \theta, x_{3}\right)$. The exponential factor $\exp (-i \omega t)$ is implied but omitted everywhere.
The exact scattered field generated when a plane elastic wave irradiates a stress-free half-plane crack is expressed by Achenbach $^{6}$ as an angular spectral decomposition. The scattered field at the observation point $\mathbf{u}_{\beta}^{\alpha}(\mathbf{x})$ can be rewritten as the Sommerfeld integral

$$
\begin{aligned}
\mathbf{u}_{\beta}^{\alpha}(\mathbf{x})= & e^{i k_{\alpha} \cos \Omega_{\alpha} x_{3}} \int_{\Gamma} f_{\beta}\left[-q_{\beta} \cos \lambda, \operatorname{sgn}(\sin \theta)\right] e^{i \zeta \cos (\lambda-\bar{\theta})} \\
& \times \mathbf{d}_{\beta}\left[-q_{\beta} \cos \lambda, \operatorname{sgn}(\sin \theta)\right] d \lambda
\end{aligned}
$$

where $f_{\beta}\left[-q_{\beta} \cos \lambda, \operatorname{sgn}(\sin \theta)\right]$ is

$$
\begin{aligned}
f_{\beta}\left[-q_{\beta} \cos \lambda, \operatorname{sgn}(\sin \theta)\right] \\
\quad=i \frac{q_{\beta} \kappa_{\beta}}{2 \pi} \sin \lambda \frac{g_{\beta}\left[-q_{\beta} \cos \lambda, \operatorname{sgn}(\sin \theta)\right]}{q_{\alpha} \cos \theta_{\alpha}-q_{\beta} \cos \lambda},
\end{aligned}
$$

with the subscript $\beta$ denoting the scattered wave mode, $q_{\beta}=k_{\beta} \sin \Omega_{\beta}, \kappa_{\beta}=c_{L} / c_{\beta}$-the dimensionless slowness of the scattered wave, and $g_{\beta}\left[-q_{\beta} \cos \lambda, \operatorname{sgn}(\sin \theta)\right]$, the numerator of the scattered wave potential being an analytical function defined in Ref. 6 (see sections 5.1 to 5.4 for definition of all the involved terms). The integrand $f_{\beta}\left[-q_{\beta} \cos \lambda\right.$, $\operatorname{sgn}(\sin \theta)]$ has then two poles $\lambda=\theta_{\beta}$ and $\lambda=2 \pi-\theta_{\beta}$ where $\theta_{\beta} \in[0, \pi]$ is defined as

$$
q_{\beta} \cos \theta_{\beta}=q_{\alpha} \cos \theta_{\alpha} .
$$

In Eq. (2), $\zeta$ is the far-field parameter

$$
\zeta=k_{\beta} L_{\beta},
$$

where $L_{\beta}$, the distance parameter, can be written as

$$
L_{\beta}=r \sin \Omega_{\beta},
$$

with $\Omega_{\beta}$ being the angle of the diffraction cone (see Fig. 1) linked to the incidence angle $\Omega_{\alpha}$ by the Snell's law $k_{\beta} \cos \Omega_{\beta}=k_{\alpha} \cos \Omega_{\alpha} ; \bar{\theta}$ is related to the position vector $\mathbf{x}$ (see Fig. 1) by

$$
\left\{\begin{array}{ll}
\bar{\theta}=\theta & \text { if } 0 \leq \theta \leq \pi \\
\bar{\theta}=2 \pi-\theta & \text { if } \pi \leq \theta \leq 2 \pi
\end{array} ;\right.
$$

$\mathbf{d}_{\beta}$ is the polarization vector of the scattered wave and $\Gamma$ is the integration contour in the complex $\lambda$-plane depicted in Fig. 2. The resulting total field is

$$
\mathbf{u}^{\mathrm{tot}}(\mathbf{x})=\mathbf{u}^{\alpha}(\mathbf{x})+\sum_{\beta} \mathbf{u}_{\beta}^{\alpha}(\mathbf{x}) .
$$


In the high frequency approximation, $\zeta \gg 1$, the integral Eq. (2) can be evaluated asymptotically. The main contributions are due to the integral critical points such as singularities of the amplitude (poles or branch points) and the stationary points of the phase function. The contributions are easy to calculate in the so-called geometrical regions where the critical points are isolated (far apart), so that the resulting asymptotics are non-uniform. The only integrand singularities considered below are the poles.

\section{A. Contributions of isolated poles: GE}

To evaluate the integral Eq. (2) asymptotically, the integration contour $(\Gamma)$ is deformed to the steepest descent contour $(\gamma)$ which passes through the phase stationary point $\bar{\theta} \in[0, \pi]$. Among the two poles $\lambda=\theta_{\beta}$ and $\lambda=2 \pi-\theta_{\beta}$, only the isolated pole $\lambda=\theta_{\beta}$ (with $0 \leq \theta_{\beta} \leq \pi$ ) can lead to a non-zero contribution to the integral (2) since it might be crossed when deforming the contour. It is only crossed when

$$
0 \leq \bar{\theta} \leq \theta_{\beta} \quad \text { (see Fig. 2) }
$$

Therefore, its contribution must be taken into account for the asymptotic evaluation of the integral Eq. (2). The GE field is the sum of this pole contribution and of the incident field. Combining Eqs. (7) and (9), this pole $\lambda=\theta_{\beta}$ gives rise to two different physical contributions depending on the observation angle $\theta$. When $\bar{\theta}=2 \pi-\theta$ [i.e., when $\pi$ $\leq \theta \leq 2 \pi$ according to (7)], the pole $\lambda=\theta_{\beta}$ is only crossed when $2 \pi-\theta_{\beta} \leq \theta \leq 2 \pi$ and its contribution then leads to the reflected field. For $\bar{\theta}=\theta$, the pole $\theta_{\beta}$ is crossed when $0 \leq \theta \leq \theta_{\beta}$. It gives rise to the so-called compensating field. This cancels the incident field when $\beta=\alpha$ and is zero otherwise. Equation (9) ensuring non-zero pole contribution is checked when the observation point lies in irradiated areas of the GE field: i.e., in the insonified zone of the reflected field $\left(2 \pi-\theta_{\beta} \leq \theta \leq 2 \pi\right.$ and $\left.\bar{\theta}=2 \pi-\theta\right)$ and in that of the compensating field $\left(0 \leq \theta \leq \theta_{\beta}\right.$ and $\left.\bar{\theta}=\theta\right)$. Then, applying the residue theorem to integral Eq. (2) and using the clockwise contour around the pole (see Fig. 2), give the amplitudes of the reflected and compensating fields,

$$
\begin{aligned}
& \lim _{\substack{\lambda \rightarrow \theta_{\beta} \\
\theta \in\left[2 \pi-\theta_{\beta}, 2 \pi\right]}}-2 \pi i f_{\beta}\left[-q_{\beta} \cos \lambda, \operatorname{sgn}(\sin \theta)\right]\left(\lambda-\theta_{\beta}\right)=\kappa_{\beta} g_{\beta}\left(-q_{\beta} \cos \theta_{\beta},-1\right)=R_{\beta}^{\alpha}, \\
& \lim _{\substack{\lambda \rightarrow \theta_{\beta} \\
\theta \in\left[0, \theta_{\beta}\right]}}-2 \pi i f_{\beta}\left[-q_{\beta} \cos \lambda, \operatorname{sgn}(\sin \theta)\right]\left(\lambda-\theta_{\beta}\right)=\kappa_{\beta} g_{\beta}\left(-q_{\beta} \cos \theta_{\beta}, 1\right)=-1, \quad \alpha=\beta, \\
& \lim _{\substack{\lambda \rightarrow \theta_{\beta} \\
\theta \in\left[0, \theta_{\beta}\right]}}-2 \pi i f_{\beta}\left[-q_{\beta} \cos \lambda, \operatorname{sgn}(\sin \theta)\right]=\kappa_{\beta} g_{\beta}\left(-q_{\beta} \cos \theta_{\beta}, 1\right)=0, \quad \alpha \neq \beta,
\end{aligned}
$$

where $R_{\beta}^{\alpha}$ is the reflection coefficient in terms of displacement. Therefore, adding the incident field to the poles contribution results in the Geometrico-Elastodynamic field

$$
\mathbf{u}^{(\mathrm{GE})}(\mathbf{x})=H\left(\eta_{\alpha}\right) \mathbf{u}^{\alpha}(\mathbf{x})+\sum_{\beta} H\left(\eta_{\beta}\right) \mathbf{u}_{\beta}^{\alpha(\mathrm{ref})}(\mathbf{x}),
$$

where $H($.$) is the Heaviside function, and where$

$$
\mathbf{u}_{\beta}^{\alpha(\mathrm{ref})}(\mathbf{x})=A R_{\beta}^{\alpha} \mathbf{d}_{\beta}\left(-q_{\beta} \cos \theta_{\beta},-1\right) e^{i k_{\beta} \mathbf{p}_{\beta} \cdot \mathbf{x}}
$$

is the reflected field; the compensating field has been combined with the incident field to produce the first term of Ref. 13. In Eq. (14), $\mathbf{p}_{\beta}=\left(\sin \Omega_{\beta} \cos \theta_{\beta},-\sin \Omega_{\beta} \sin \theta_{\beta}, \cos \Omega_{\beta}\right)$ is the unit reflected wave vector and $\mathbf{d}_{\beta}\left(-q_{\beta} \cos \theta_{\beta},-1\right)$ its polarisation vector. The arguments $\eta_{\alpha}=\operatorname{sgn}\left(\theta-\theta_{\alpha}\right)$ and $\eta_{\beta}$ $=\operatorname{sgn}\left(\theta-2 \pi+\theta_{\beta}\right)$ of the respective Heaviside functions determine whether the observation point is in the illuminated region or shadow of incident and reflected waves (see Fig. 3).

\section{B. Contributions of isolated stationary points: GTD}

The contribution of each isolated stationary point $\lambda_{s}=\bar{\theta}$ can be found by applying the steepest descent method to Eq. (2), producing the classical GTD recipe

$$
\begin{aligned}
\mathbf{u}_{\beta}^{\alpha(\mathrm{GTD})}(\mathbf{x})= & u^{\alpha}\left(\mathbf{x}_{\beta}^{\alpha}\right) D_{\beta}^{\alpha(\mathrm{GTD})}\left(\Omega_{\alpha}, \theta_{\alpha}, \theta\right) \\
& \times \mathbf{d}_{\beta}\left[-q_{\beta} \cos \theta, \operatorname{sgn}(\sin \theta)\right] \frac{\mathrm{e}^{i k_{\beta} S_{\beta}}}{\sqrt{k_{\beta} L_{\beta}}},
\end{aligned}
$$

where $u^{\alpha}\left(\mathbf{x}_{\beta}^{\alpha}\right)=\mathbf{u}^{\alpha}\left(\mathbf{x}_{\beta}^{\alpha}\right) \cdot \mathbf{d}^{\alpha}, \mathbf{x}_{\beta}^{\alpha}=\left(0,0, x_{3}-S_{\beta} \cos \Omega_{\beta}\right) \quad$ is the diffraction point on the scattering edge (see Fig. 1); $S_{\beta}$ is the distance between the diffraction point $\mathbf{x}_{\beta}^{\alpha}$ and the observation point $\mathbf{x}$. Also,

$$
\begin{aligned}
& D_{\beta}^{\alpha(\mathrm{GTD})}\left(\Omega_{\alpha}, \theta_{\alpha}, \theta\right) \\
& \quad=\frac{q_{\beta} \kappa_{\beta}}{\sqrt{2 \pi}} \frac{g_{\beta}\left[-q_{\beta} \cos \theta, \operatorname{sgn}(\sin \theta)\right]}{q_{\alpha} \cos \theta_{\alpha}-q_{\beta} \cos \theta}|\sin \theta| e^{i \pi / 4}
\end{aligned}
$$

are GTD diffraction coefficients. They contain poles $\theta=\theta_{\beta}$ and $\theta=2 \pi-\theta_{\beta}$, which describe the shadow boundaries (see Fig. 3).

Note that at the incident and reflected shadow boundaries the total GE field Eq. (13) is finite while the GTD diffracted field is infinite. Therefore, the approximate GTDbased total field 


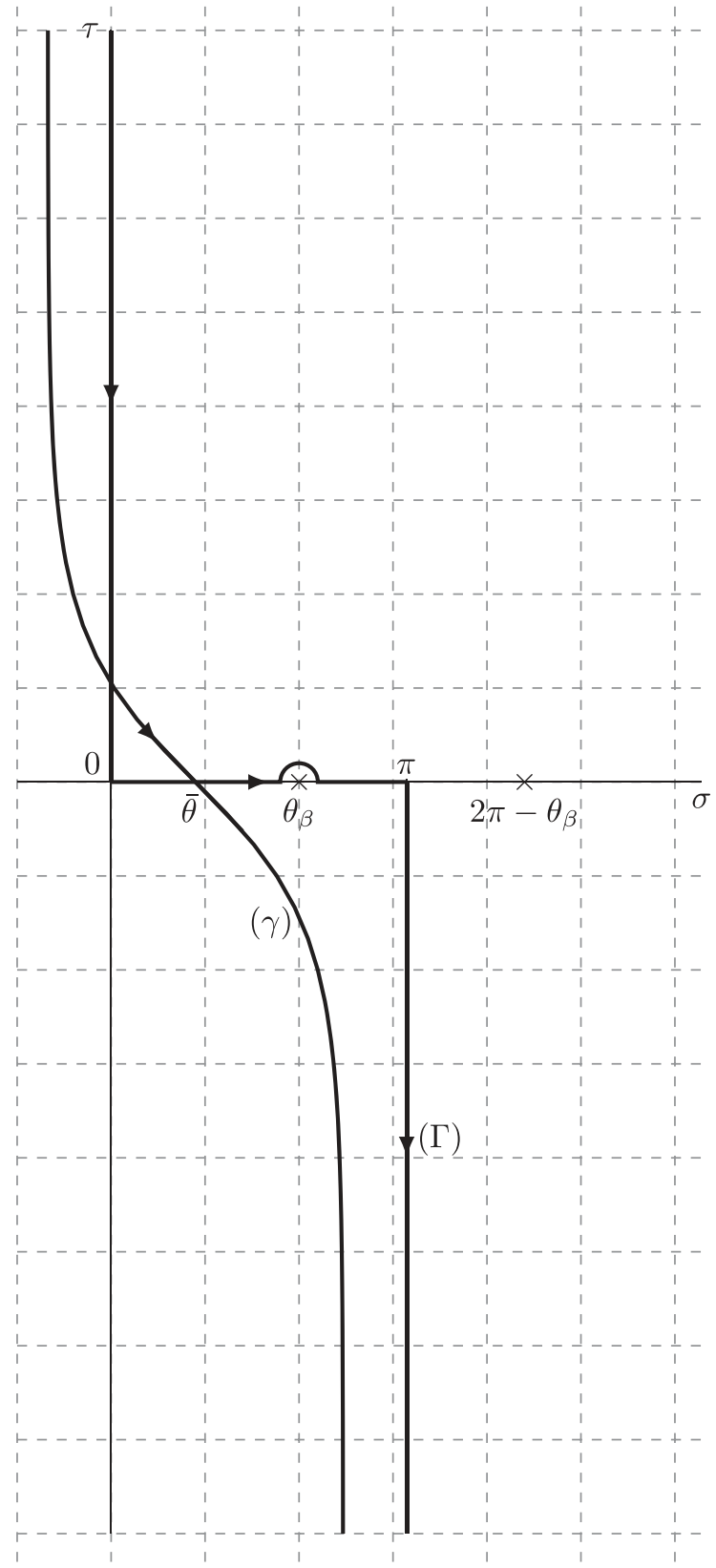

FIG. 2. $\Gamma$ and the steepest descent path $\gamma$ in the complex plane $\lambda=\sigma+\tau . \theta_{\beta}$ is the real pole of the integral [Eq. (2)] and $\bar{\theta}$ is its phase stationary point.

$$
\mathbf{u}^{\mathrm{tot}(\mathrm{GTD})}(\mathbf{x})=\mathbf{u}^{(\mathrm{GE})}(\mathbf{x})+\sum_{\beta} \mathbf{u}_{\beta}^{\alpha(\mathrm{GTD})}(\mathbf{x}),
$$

is not spatially uniform.

\section{SCATTERING OF A PLANE ELASTIC WAVE BY A HALF-PLANE CRACK: UTD}

To overcome the non-uniformity of the approximate total field Eq. (17), UTD is derived in this section in elastodynamics. When the phase stationary point $\lambda_{s}=\bar{\theta}$ in Eq. (2) coalesces with the simple pole $\lambda=\lambda_{0}(\tau)=\theta_{\beta}\left(\theta_{\beta} \leq \pi\right)$, Eq. (2) can be approximated using the Pauli-Clemmow procedure. ${ }^{18,19}$ Note that the integral Eq. (2) has the form

$$
I(\zeta, \tau)=\int_{\gamma} h(\lambda, \tau) e^{\zeta q(\lambda)} d \lambda
$$

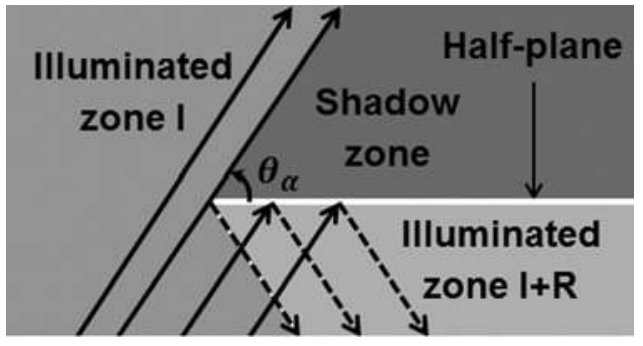

FIG. 3. Interaction of a plane wave with a semi-infinite crack embedded in an elastic homogeneous solid. Thick arrows, incident waves; dash arrows, reflected waves.

where $\zeta \gg 1$ and $\tau$ is a complex-valued parameter, $h(\lambda, \tau)$ and $q(\lambda)$ are $\lambda$ holomorphic functions, and $\gamma$ is the steepest descent path. Note too that $\bar{\theta}$ cannot coalesce with the simple pole $\lambda=2 \pi-\theta_{\beta}$, because $\bar{\theta}$ is always smaller than $\pi$, while $2 \pi$ $-\theta_{\beta}$ is always larger than $\pi$. Therefore the Pauli-Clemmow approximation to the scattered field is

$$
\mathbf{u}_{\beta}^{\alpha(\mathrm{UTD})}=F\left(k_{\beta} L_{\beta} a\right) \mathbf{u}_{\beta}^{\alpha(\mathrm{GTD})}
$$

where $a$ is the phase difference between the rays propagating in direction $\bar{\theta}$ and $\theta_{\beta}$

$$
a=2 \sin ^{2}\left(\frac{\theta_{\beta}-\bar{\theta}}{2}\right),
$$

and $\mathrm{F}$ is a transition function

$$
F(X)=-2 i \sqrt{X} \sqrt{i \pi} e^{-i X} \bar{F}(\sqrt{X}), \quad-\frac{\pi}{2}<\arg X<\frac{3 \pi}{2},
$$

with $\bar{F}$ being the Fresnel function,

$$
\bar{F}(X)=\frac{1}{\sqrt{i \pi}} \int_{X}^{+\infty} e^{i t^{2}} d t
$$

The transition function is the complex conjugate of the Kouyoumjian function. ${ }^{12}$ Due to the presence of $\sqrt{X}, F(X)$ is multivalued and can be rendered single-valued in a standard manner, using, e.g., the branch cut along the negative imaginary axis $\{\operatorname{Im} X<0, \operatorname{Re} X=0\}$.

Using Eq. (19), a UTD diffraction coefficient can be defined as

$$
D_{\beta}^{\alpha(\mathrm{UTD})}=F\left(k_{\beta} L_{\beta} a\right) D_{\beta}^{\alpha(\mathrm{GTD})}
$$

Substituting Eqs. (15) and (23) in Eq. (19), the UTD diffracted field can be expressed as

$$
\begin{aligned}
\mathbf{u}_{\beta}^{\alpha(\mathrm{UTD})}= & u^{\alpha}\left(\mathbf{x}_{\beta}^{\alpha}\right) D_{\beta}^{\alpha(\mathrm{UTD})} \frac{\exp \left(i k_{\beta} S_{\beta}\right)}{\sqrt{k_{\beta} L_{\beta}}} \\
& \times \mathbf{d}_{\beta}\left[-q_{\beta} \cos \theta, \operatorname{sgn}(\sin \theta)\right] .
\end{aligned}
$$

It has the same form as the GTD diffracted field and the UTD diffraction coefficient differs from the GTD one by the transition function. When the observation point is far from the shadow boundaries the approximation Eq. (24) is 
equivalent to GTD. Indeed, the asymptotic value of the Fresnel function in the illuminated region given by Borovikov $^{20}$ permits to find

$$
F\left(k_{\beta} L_{\beta} a\right) \underset{\left|\bar{\theta}-\theta_{\beta}\right| \rightarrow+\infty}{\longrightarrow} 1 .
$$

Since $\bar{F}(0)=1 / 2$, near the shadow boundaries,

$$
F\left(k_{\beta} L_{\beta} a\right) \sim e^{-i(\pi / 4)}(\operatorname{sgn} \epsilon) \epsilon \sqrt{\frac{\pi}{2}} \sqrt{k_{\beta} L_{\beta}},
$$

where $\epsilon=\bar{\theta}-\theta_{\beta}$ is a small number $(|\epsilon| \ll 1)$. One can use Eqs. (26), (10)-(12) in the asymptotics of Eq. (23) to obtain

$$
D_{\beta}^{\alpha(\text { UTD })} \sim \begin{cases}0 & \text { if } \theta \leq \pi \text { and } \alpha \neq \beta \\ -\frac{\operatorname{sgn} \epsilon}{2} \sqrt{k_{\beta} L_{\beta}}, & \text { if } \theta \leq \pi \text { and } \alpha=\beta \\ \frac{R_{\beta}^{\alpha}}{2}(\operatorname{sgn} \epsilon) \sqrt{k_{\beta} L_{\beta}}, & \text { if } \theta>\pi .\end{cases}
$$

The UTD diffraction coefficients do not diverge at shadow boundaries as the GTD coefficients do, however the sign function makes them discontinuous. It follows that near the shadow boundaries, the leading-order asymptotics of the diffracted field Eq. (24) are

$$
\mathbf{u}_{\beta}^{\alpha(\mathrm{UTD})}(\mathbf{x}) \sim \begin{cases}0, & \text { if } \theta \leq \pi \text { and } \alpha \neq \beta \\ -A \frac{\operatorname{sgn} \epsilon}{2} e^{i k_{\alpha} \cos \Omega_{\alpha}\left(x_{3}-S_{\alpha} \cos \Omega_{\alpha}\right)} e^{i k_{\alpha} S_{\alpha}} \mathbf{d}^{\alpha}, & \text { if } \theta \leq \pi \text { and } \alpha=\beta \\ A \frac{R_{\beta}^{\alpha}}{2}(\operatorname{sgn} \epsilon) e^{i k_{\alpha} \cos \Omega_{\alpha}\left(x_{3}-S_{\beta} \cos \Omega_{\beta}\right)} e^{i k_{\beta} S_{\beta}} \mathbf{d}_{\beta}\left(-q_{\beta} \cos \theta_{\beta},-1\right), & \text { if } \theta>\pi,\end{cases}
$$

where the exponential term $\exp \left[i k_{\alpha} \cos \Omega_{\alpha}\left(x_{3}-S_{\alpha, \beta} \cos \Omega_{\alpha, \beta}\right)\right]$ is the phase of the incident wave at the diffraction point $\mathbf{x}_{\beta}^{\alpha}$. Near the incident shadow boundary, when $\alpha \neq \beta$ the UTD diffracted field does not exist and due to the presence of the sign function when $\alpha=\beta$, it is discontinuous. The UTD diffracted field is discontinuous at the reflected shadow boundary too.

However, since the approximation UTD Eq. (24) works in the vicinity of shadow boundaries and describes diffracted fields outside them, adding to it the GE field produces the UTD approximation to the total field,

$$
\mathbf{u}^{\mathrm{tot}(\mathrm{UTD})}(\mathbf{x})=\mathbf{u}_{\beta}^{\alpha(\mathrm{GE})}(\mathbf{x})+\sum_{\beta} \mathbf{u}_{\beta}^{\alpha(\mathrm{UTD})}(\mathbf{x}) .
$$

It is easy to show that this approximate total field is continuous. Indeed, since $r=S_{\alpha, \beta} \sin \Omega_{\alpha, \beta}$, Eq. (13) can be rewritten as

$$
\begin{aligned}
\mathbf{u}^{\mathrm{GE}}(\mathbf{x})= & A\left(H\left(\eta_{\alpha}\right) e^{i k_{\alpha}\left[S_{\alpha}+\cos \Omega_{\alpha}\left(x_{3}-S_{\alpha} \cos \Omega_{\alpha}\right)\right] \cos \left(\theta-\theta_{\alpha}\right)}\right. \\
& \times e^{i k_{\alpha} \cos \Omega_{\alpha} x_{3}\left(1-\cos \left(\theta-\theta_{\alpha}\right)\right)} \mathbf{d}^{\alpha}+H\left(\eta_{\beta}\right) R_{\beta}^{\alpha} \\
& \times e^{i k_{\beta}\left[S_{\beta}+\cos \Omega_{\beta}\left(x_{3}-S_{\beta} \cos \Omega_{\beta}\right)\right] \cos \left(\theta+\theta_{\beta}\right)} \\
& \left.\times e^{i k_{\alpha} \cos \Omega_{\beta} x_{3}\left[1-\cos \left(\theta+\theta_{\beta}\right)\right]} \mathbf{d}_{\beta}\left(-q_{\beta} \cos \theta_{\beta},-1\right)\right),
\end{aligned}
$$

where the arguments of the Heaviside functions are, respectively,

$$
\begin{aligned}
& \eta_{\alpha}=\operatorname{sgn}\left(\theta-\theta_{\alpha}\right) \\
& \eta_{\beta}=\operatorname{sgn}\left(\theta-2 \pi+\theta_{\beta}\right) .
\end{aligned}
$$

Therefore near the shadow boundaries, the GE filed can be approximated using

$$
\mathbf{u}^{\mathrm{GE}}(\mathbf{x}) \approx \begin{cases}A e^{i k_{\alpha}\left[S_{\alpha}+\cos \Omega_{\alpha}\left(x_{3}-S_{\alpha} \cos \Omega_{\alpha}\right)\right]} H(\epsilon) \mathbf{d}^{\alpha} & \text { if } \theta \leq \pi \\ A R_{\beta}^{\alpha} e^{i k_{\beta}\left[S_{\beta}+\cos \Omega_{\beta}\left(x_{3}-S_{\beta} \cos \Omega_{\beta}\right)\right]} H(-\epsilon) \mathbf{d}_{\beta}\left(-q_{\beta} \cos \theta_{\beta},-1\right)+\mathbf{u}^{\alpha}\left(-\theta_{\alpha}\right) & \text { if } \theta>\pi\end{cases}
$$

Finally, expressing the Heaviside function as

$$
H(\epsilon)=\frac{1}{2}(1+\operatorname{sgn} \epsilon)
$$

it can be seen that near both the incident and reflected shadow boundaries the GE field (13) is discontinuous and its discontinuities cancel those of the UTD diffracted field. Thus, the UTD total field Eq. (29) is continuous.
The contribution of coalescing stationary phase point and pole can also be calculated by UAT (Ref. 6) (see Appendix). It is well understood in electromagnetism ${ }^{21}$ that unlike UAT, the UTD asymptotics do not include all terms of the order $\left(k_{\beta} L_{\beta}\right)^{-(1 / 2)}$.

\section{COMPARISON OF UTD AND UAT AND DISCUSSION}

Let us first discuss the difference in analytical properties of various UTD and UAT fields. When comparing the UTD 
(a)

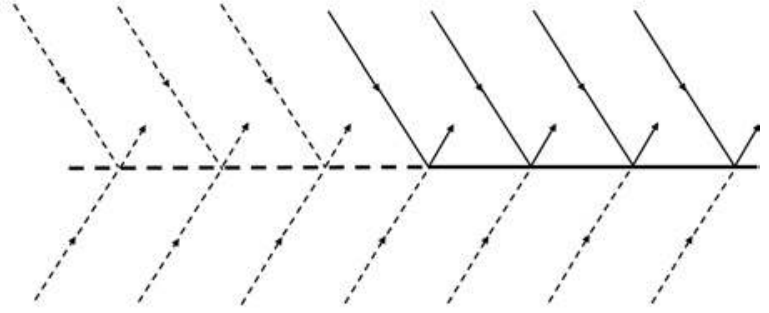

(b)

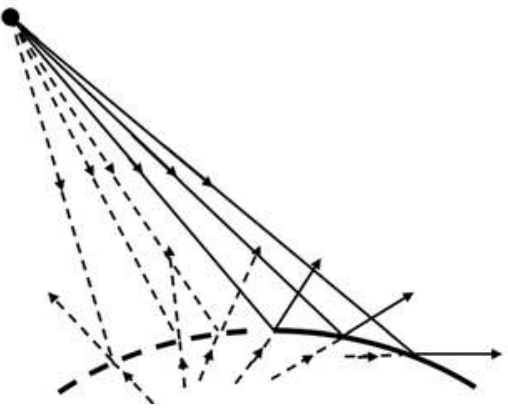

FIG. 4. Extension of the reflected field to its shadow zone using fictitious rays (a) half-plane (thick line) illuminated by a plane wave and- (b) curved face (thick line) illuminated by a spherical wave.

total field Eq. (29) to the GTD-based non-uniform approximation Eq. (17), the GE field is unchanged while the diffraction coefficient is multiplied by the transition function Eq. (21). The argument $k_{\beta} L_{\beta} a$ of the transition function describes the proximity of the observation point to a shadow boundary. When the observation point moves away from such boundary $k_{\beta} L_{\beta} a$ increases, the transition function approaches 1 [see Eq. (25)] and UTD diffraction coefficient approaches the GTD diffraction coefficient. When the observation point moves towards such boundary, $k_{\beta} L_{\beta} a$ and the transition function both vanish but as shown in Sec. III the UTD coefficient is discontinuous and the UTD total field continuous.

By contrast, when UAT is presented in the form Eq. (A1), it is the diffracted field that remains the same as in the GTD-based non-uniform approximation Eq. (17), while the GE field is modified. In UAT both the modified GE fields and GTD fields diverge at shadow boundaries, but the corresponding singularities cancel each other, and the total UAT field is again continuous. Since in Eq. (A1) the incident and reflected fields are defined on the whole space, both incident and reflected fields have to be extended to their corresponding shadow region. This can be achieved by tracing the incident rays through the obstacle as if it was absent. Moreover, the reflected field has to be extended to the shadow region of the reflected field too. This can be achieved by extending the illuminated face beyond the crack edge and tracing the resulting fictitious reflected rays (see Fig. 4).

While reliance on the fictitious rays is a definite disadvantage, it has been mentioned above that, unlike UTD, UAT is more consistent in that it contains all terms of order $\left(k_{\beta} L_{\beta}\right)^{-(1 / 2)}$. In electromagnetism, the terms missing in UTD are small. Therefore we move on to a numerical comparison of UTD and UAT to establish whether the same is true in elastodynamics. Note that the elastodynamic UAT has been tested before, using the finite difference numerical method (see section 2.3.1 in Ref. 22). (a)
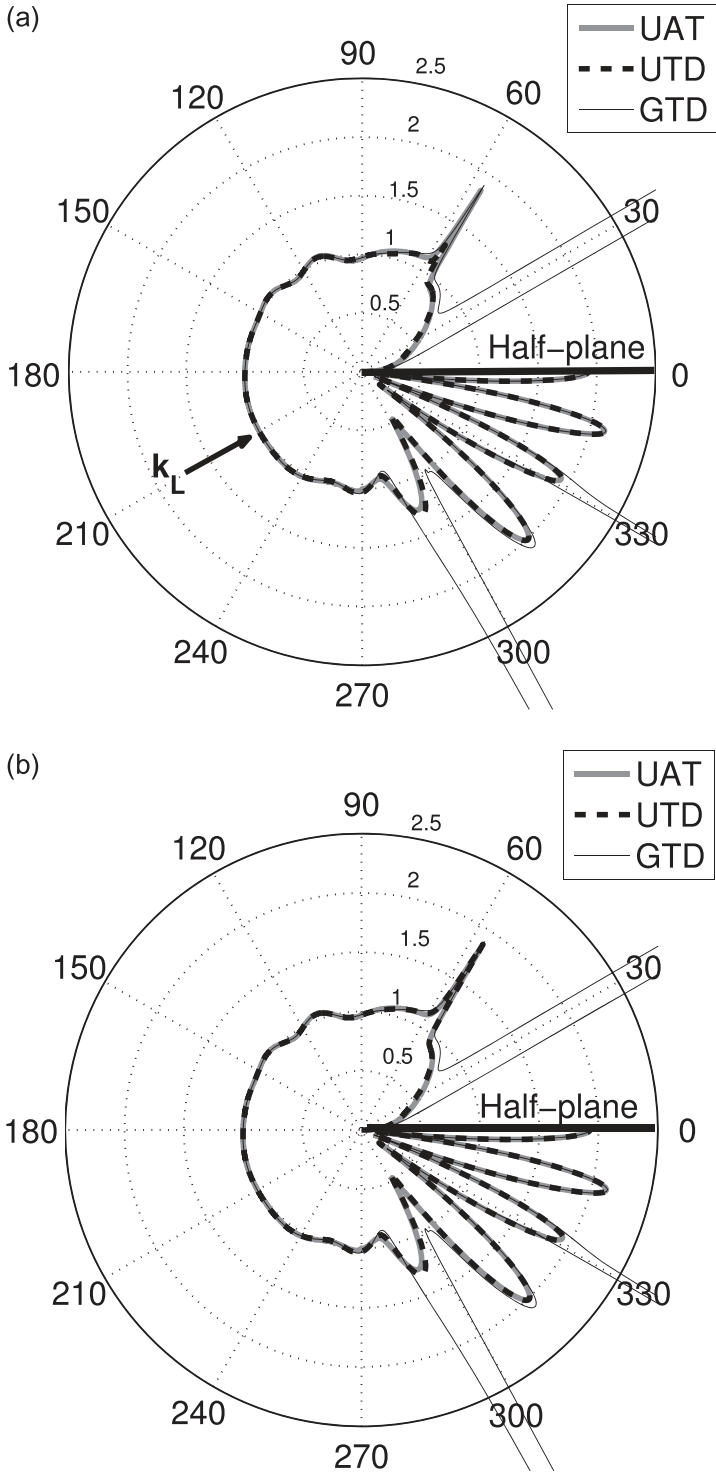

FIG. 5. Directivity pattern of the total field predicted by different models (GTD, UAT, and UTD) at $r=2 \lambda_{L} ; \Omega_{L}=90^{\circ}, \theta_{L}=30^{\circ}$. (a) UTD model is the one found using Pauli-Clemmow method. (b) Modified UTD model. Each circle represents amplitude of the total field normalized by the incident amplitude.

To compare UAT and UTD, both two-dimensional (2D) and three-dimensional (3D) configurations are considered below, with $\Omega_{\alpha}=90^{\circ}$ and $\Omega_{\alpha} \neq 90^{\circ}$, respectively. In a $2 \mathrm{D}$ configuration, the incident wave is normal to the edge crack and thus the diffracted waves have cylindrical fronts, while in a $3 \mathrm{D}$ configuration the incidence is oblique and the diffracted waves have conical fronts. The numerical results are presented in the $\left(\mathbf{e}_{1}, \mathbf{e}_{2}\right)$ plane, which is perpendicular to the edge crack, since the problem is invariant in the $x_{3}$ direction (see Fig. 1). The observation is specified using the cylindrical coordinates $(r, \theta)$ associated with the $\left(\mathbf{e}_{1}, \mathbf{e}_{2}\right)$ plane. In all simulations, the solid material is ferritic steel with Poisson's ratio $\nu=0.29$, longitudinal speed $c_{L}=5900 \mathrm{~m} \mathrm{~s}^{-1}$ and transversal speed $c_{T}=3230 \mathrm{~ms}^{-1}$.

In far field from the flaw, the scattering patterns exhibit a lot of oscillation lobes. We present first results for $r=2 \lambda_{\alpha}$ and $3 \lambda_{\alpha}$ and then at $r=8 \lambda_{\alpha}\left(\lambda_{\alpha}\right.$ being the wavelength of the 


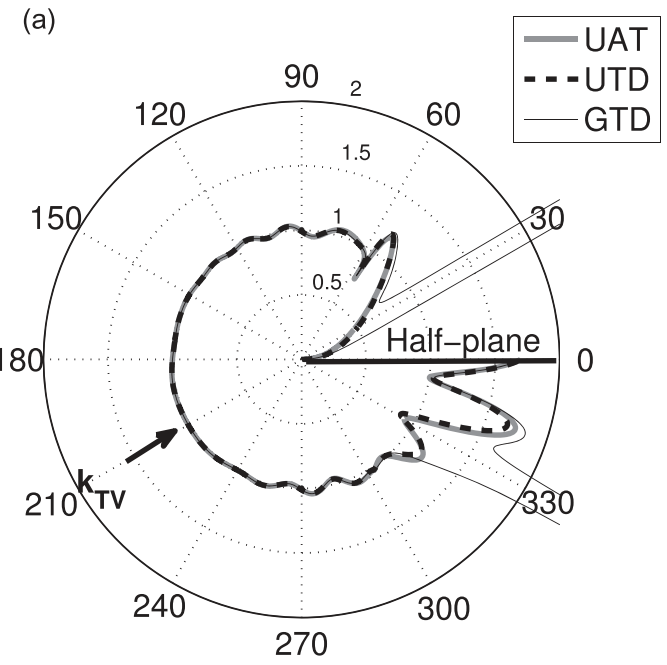

(b)

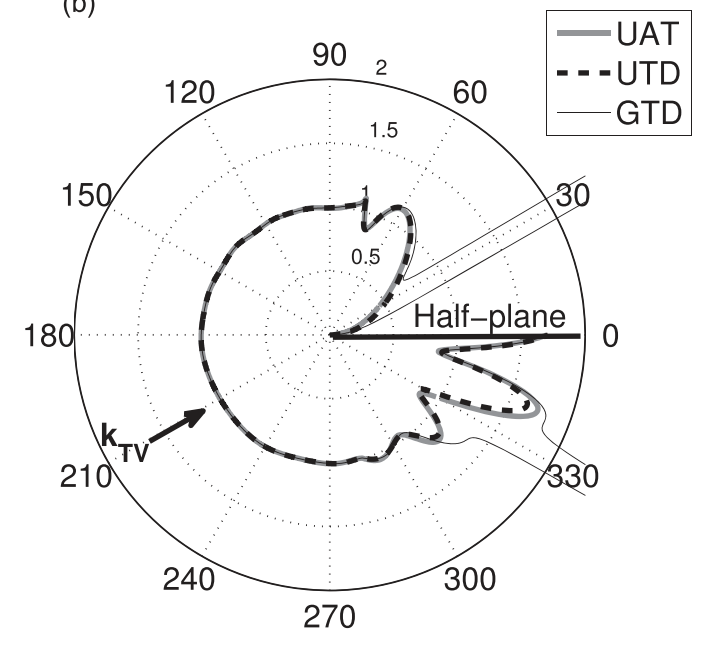

FIG. 6. Directivity pattern of the total field predicted by different models (GTD, UAT, and UTD) at $r=3 \lambda_{T V}$ for $\theta_{T V}=30^{\circ}$. (a) $\Omega_{T V}=90^{\circ}$, (b) $\Omega_{T V}=60^{\circ}$. Each circle represents amplitude of the total field normalized by the incident amplitude.

incident wave) so that the patterns have less lobes and lend themselves to a clear interpretation (see Figs. 5, 6, and 9). In Figs. 5 and 6 all approximate total fields are calculated using all scattered modes, with the GTD-based non-uniform asymptotics given by Eq. (17), UAT given by Eq. (A1), and UTD given by (29). In Figs. 5 and 6 the (a) configurations

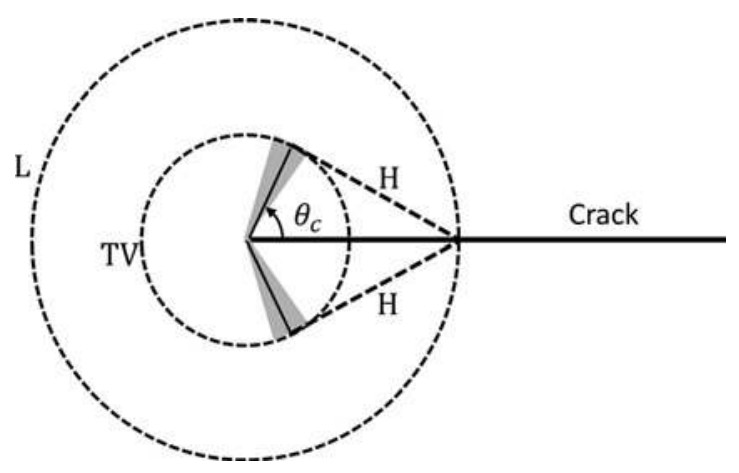

FIG. 7. Waves diffracted from a crack edge: L stands for longitudinal wave, $\mathrm{TV}$ for transversal, and $\mathrm{H}$ for head wave; $\theta_{c}$ is the longitudinal critical angle.

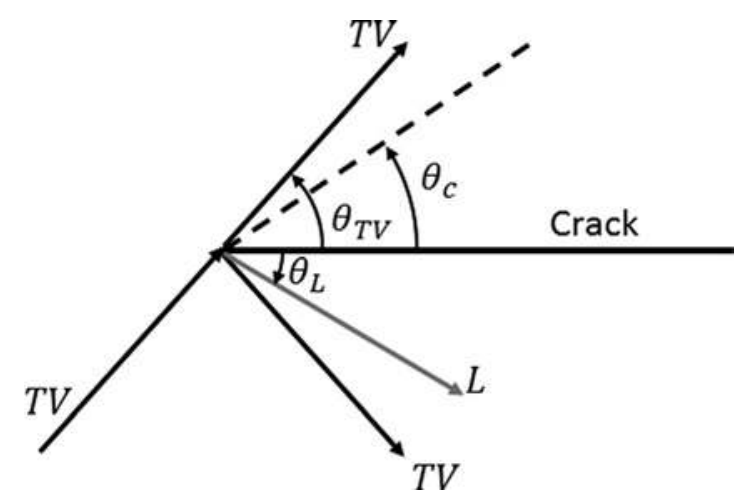

FIG. 8. Reflection of a transversal wave by a half-plane crack. An incident transversal wave gives rise to reflected transversal and longitudinal waves, which respect the Snell's law $\left(\theta_{L}<\theta_{T V}\right)$. For a transversal incidence below the longitudinal critical angle $\theta_{c}\left(\theta_{T V}<\theta_{c}\right)$, the longitudinal waves are no more reflected but laid on the crack surface.

are 2D and in Fig. 6, the (b) configuration is 3D. As expected, both UTD and UAT are continuous at shadow boundaries and practically coincide far away from the shadow boundaries. Near the shadow boundaries they differ but not by much. There are three shadow boundaries in Fig. 5, the incident $\mathrm{L}$ shadow boundary $\theta=30^{\circ}$, reflected TV shadow boundary $\theta \approx 300^{\circ}$ and reflected $\mathrm{L}$ shadow boundary $\theta=330^{\circ}$. The peaks near critical angles $\theta_{c} \approx$ $56.8^{\circ}$ and $2 \pi-\theta_{c} \approx 303,2^{\circ}$ are due to the interference of the diffracted $\mathrm{T}$ wave with the corresponding head waves generated by diffraction at the edge (see Fig. 7). The head waves are contributions to the exact solution Eq. (2) of the integrand's branch points. ${ }^{23}$ The coalescence between the stationary phase points and branch points lies outside the scope of this paper. However, it is worth noting that while GTD and its uniform corrections are not valid near the critical angle, since the head wave attenuates with the distance, in far-field the spikes observed in the diffraction coefficient $D_{\mathrm{TV}}^{\alpha}$ at the critical angles are physical in nature. In Fig. 5(a), UAT reproduces the critical GTD peak exactly, while UTD has a smaller amplitude. The discrepancy can be explained by noting that each UTD diffraction coefficient has four poles, one at the incident angle $\theta_{\alpha}$, one at the reflection angle $2 \pi-\theta_{\alpha}$, one at the reflection angle $2 \pi-\theta_{\beta}$, and one at the reflection angle $\theta_{\beta}$. When $\beta \neq \alpha, 2 \pi-\theta_{\beta}$ is the propagation angle of a mode-converted reflected wave and $\theta_{\beta}$ is an angle associated with a non-physical mode-converted transmitted wave. It is non-physical, because the residue at this pole is zero and the diffraction coefficient $D_{\beta}^{\alpha}, \beta \neq \alpha$ does not diverge (see Figs. 4.5 and 4.6 in Ref. 24). In the vicinity of this pole, the transition function tends to 0 [see Eq. (26)] and the UTD diffraction coefficient differs from the corresponding GTD diffraction coefficient. In Fig. 5(a), such pole $\theta_{T V} \approx 61.70^{\circ}$ is close to the critical angle $\theta_{c} \approx 57^{\circ}$ and therefore near this critical angle the peak amplitude is reduced. The performance of UTD can be improved by taking into account that since the residue of the pole $\theta_{\beta}, \beta \neq \alpha$ is zero and therefore there is no need to consider its coalescence with the stationary points, it is possible to introduce the following modified UTD (MUTD): 
for $\beta \neq \alpha$,

$$
\text { if } \theta_{\alpha} \leq \pi, \quad \begin{cases}D_{\beta}^{\alpha(\mathrm{UTD})}=D_{\beta}^{\alpha(\mathrm{GTD})} & \text { if } 0 \leq \theta \leq \pi \\ D_{\beta}^{\alpha(\mathrm{UTD})}=F\left(k_{\beta} L_{\beta} \sin ^{2}\left(\frac{\theta+\theta_{\beta}}{2}\right)\right) D_{\beta}^{\alpha(\mathrm{GTD})} & \text { if } \pi<\theta \leq 2 \pi\end{cases}
$$

and

$$
\text { if } \theta_{\alpha}>\pi, \quad \begin{cases}D_{\beta}^{\alpha(\mathrm{UTD})}=F\left(k_{\beta} L_{\beta} \sin ^{2}\left(\frac{\theta-\theta_{\beta}}{2}\right)\right) D_{\beta}^{\alpha(\mathrm{GTD})} & \text { if } 0 \leq \theta \leq \pi \\ D_{\beta}^{\alpha(\mathrm{UTD})}=D_{\beta}^{\alpha(\mathrm{GTD})} & \text { if } \pi<\theta \leq 2 \pi .\end{cases}
$$

The UTD curve in Fig. 5(b) confirms that the Modified UTD reproduces the GTD critical peaks

In Figs. 6(a) and 6(b), there are only two shadow boundaries, the incident TV shadow boundary $\theta=30^{\circ}$ and reflected TV shadow boundary $\theta=330^{\circ}$. Since the incidence angle $\theta_{\mathrm{TV}}$ is subcritical there are no reflected longitudinal waves. In the configuration used in Fig. 8 the incident angle $\theta_{\mathrm{TV}}$ is supercritical.

The simulations reported in Fig. 9 have been performed using parameters similar to Fig. 5(b) but for a larger far-field parameter $\zeta=16 \pi \sin \Omega_{\beta}$ (so that $r=8 \lambda_{L}$ ). The resulting UAT and UTD fields are much closer than in Fig. 6(b). As the observation point moves away from the crack, near the shadow boundaries the error between the approximations decreases [see Figs. 10(a), 10(b), and 10(c)]. The modified UTD produces a much smaller error near the critical angle $\theta_{c} \approx 57^{\circ}$ than UTD.

The above results are envisaged as particularly useful in NDT of cracks in materials. Indeed, UTD has already proved an efficient method for simulating the scattering of an elastic plane wave by a half-plane. In NDT, the flaws are usually

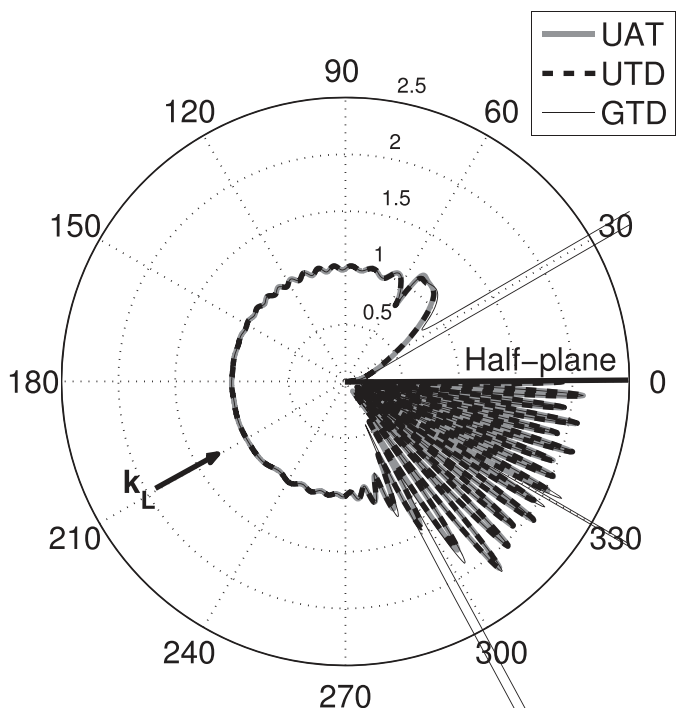

FIG. 9. Directivity pattern of the total field predicted by different models (GTD, UAT, and UTD) at $r=8 \lambda_{L} ; \Omega_{L}=90^{\circ}, \theta_{L}=30^{\circ}$. Each circle represents an amplitude of the total field normalized by the incident amplitude. inspected in the far field of the used probes or else in their focal areas if they are focused. Consequently, often, at each point of the meshed flaw the incident wave-fields can be approximated by a wave that is locally plane ${ }^{25,26}$ and GE ray methods and GTD-based models can be easily applied in the probes far field. ${ }^{27}$ Since the proposed UTD model relies on the same high-frequency assumptions as GTD, it should prove to be efficient in the far field of the flaw. If inspection is carried out in the near or intermediate field, the approximation of the locally plane wave for the incident field can be withdrawn, as has been shown in the recent report on the new promising GTD-based NDT system model which uses each incident ray as an input of the flaw scattering model. ${ }^{28}$

The proposed UTD model can be used to model scattered echoes from any locally plane flaw, since according to the GTD locality principle, a curved edge of the flaw contour can be approximated by the edge of the half-plane, which is tangent to it. In modelling specular reflections, the finite size of the flaw is routinely accounted for in the GE ray method: only the incident rays impacting on the finite flaw surface are reflected. In modelling edge diffraction, the finite extent of the edge is accounted for by using incremental methods. ${ }^{29}$ The limits of validity of GTD-based NDT system models are described, e.g., in Ref. 27 for a rectangular flaw. It has been shown that GTD predictions are valid for flaw heights of more than one wavelength for longitudinal waves and more than two wavelengths for shear waves (whether incident and diffracted). The NDT models based on UTD can be extended to employ solutions of canonical problems of wedge diffraction to model 3D multi-facetted or branched flaws (which comprise planar facets).

\section{CONCLUSIONS}

The elastodynamic UTD has been derived to describe the scattering of a plane wave from a stress-free half-plane. Unlike GTD, at the shadow boundaries, each UTD diffracted field contains no singularities, only discontinuities, and each such discontinuity cancels the discontinuity in the corresponding GE field. Just like the total UAT field, the total UTD field is continuous. In the far field UTD practically coincides with UAT and the modified version of UTD, 

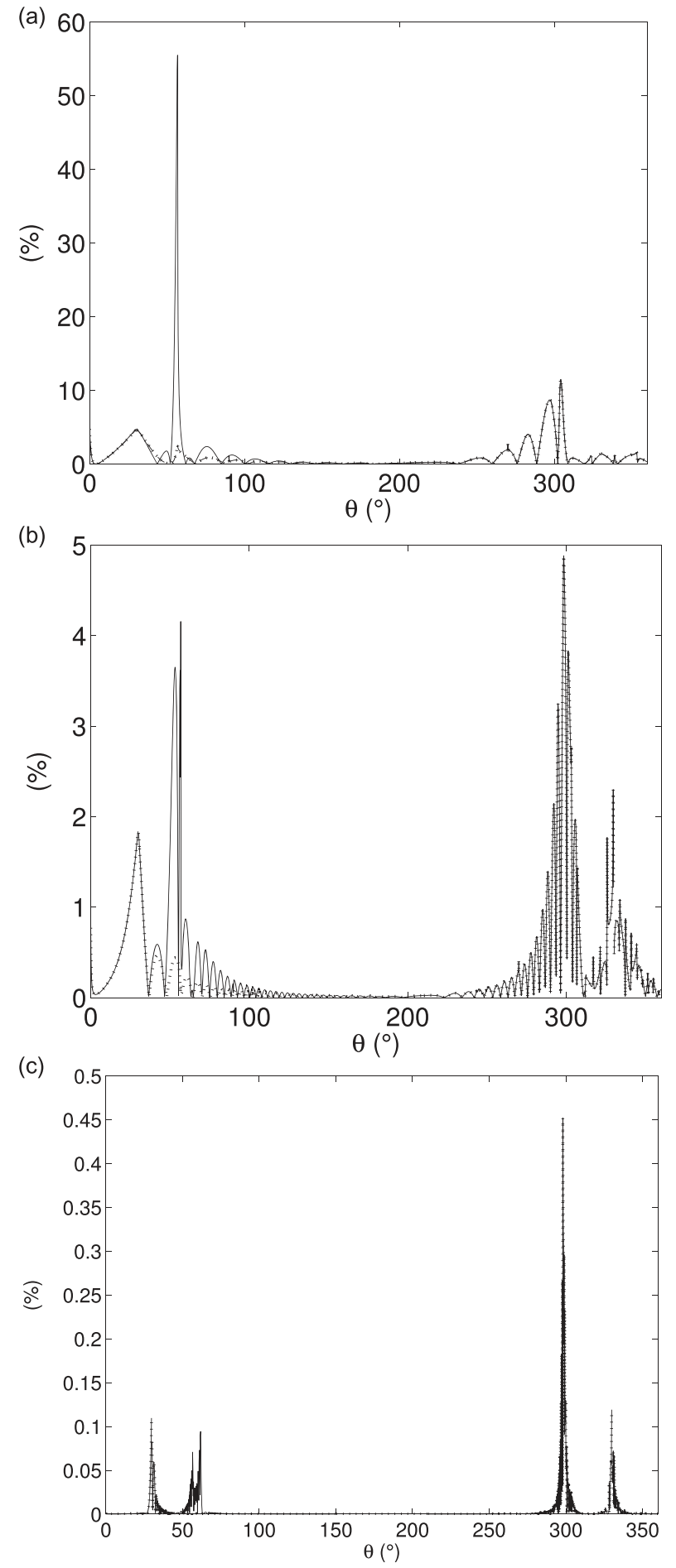

FIG. 10. Absolute error between UTD and UAT total fields in percentage of the incident displacement amplitude [noted $A$ in Eq. (1)] versus the observation angle at $\Omega_{L}=90^{\circ}, \theta_{L}=30^{\circ}$ in percent of the incident amplitude (a) $r=2 \lambda_{L}$, (b) $r=8 \lambda_{L}$, (c) $r=500 \lambda_{L}$. Solid line represents the absolute error between initial UTD and UAT total fields and dashed line represents absolute error between modified UTD and UAT. (a), (b), and (c) use different scales along the vertical axis.

MUTD, proposed in this paper, reproduces the critical peaks in UAT too.

In ray tracing implementations UTD is more convenient than UAT, because unlike UAT, it does not rely on fictitious rays. It is well understood that, unlike UAT, UTD misses terms of the same order it includes, however the numerical comparisons carried out in this paper confirm that near the shadow boundaries, where the missing terms play a part, the resulting differences do not appear to be significant for practical applications (see, for example, Ref. 30).

\section{APPENDIX: THE ELASTODYNAMIC UAT}

The total field in elastodynamic UAT can be presented in different ways. Here we present it in the same form as in Ref. 6,

$$
\begin{aligned}
\mathbf{u}^{\mathrm{tot}(\mathrm{UAT})}(\mathbf{x})= & A\left[\bar{F}\left(\xi_{\alpha}\right)-\hat{\bar{F}}\left(\xi_{\alpha}\right)\right] e^{i \mathbf{k}^{\alpha} \cdot \mathbf{x}} \mathbf{d}^{\alpha} \\
& +\sum_{\beta} A R_{\beta}^{\alpha}\left[\bar{F}\left(\xi_{\beta}\right)-\hat{\bar{F}}\left(\xi_{\beta}\right)\right] e^{i k_{\beta} \mathbf{p}_{\beta} \cdot \mathbf{x}} \\
& \times \mathbf{d}_{\beta}\left[q_{\beta} \cos \theta_{\beta}, \operatorname{sgn}(\sin \theta)\right] \\
& +\sum_{\beta} u^{\alpha}\left(\mathbf{x}_{\beta}^{\alpha}\right) D_{\beta}^{\alpha(\mathrm{GTD})} \frac{e^{i k_{\beta} S_{\beta}}}{\sqrt{k_{\beta} L_{\beta}}} \\
& \times \mathbf{d}_{\beta}\left[q_{\beta} \cos \theta, \operatorname{sgn}(\sin \theta)\right]
\end{aligned}
$$

where

$$
\hat{\bar{F}}(X)=e^{i(\pi / 4)} \frac{e^{i X^{2}}}{2 X \sqrt{\pi}},
$$

and the parameters

$$
\begin{aligned}
& \xi_{\alpha}=-\operatorname{sgn}\left(\theta-\theta_{\alpha}\right) \sqrt{2 k_{\alpha} L_{\alpha} \sin ^{2}\left(\frac{\theta_{\alpha}-\theta}{2}\right)} \text { and } \\
& \xi_{\beta}=-\operatorname{sgn}\left(\theta+\theta_{\beta}-2 \pi\right) \sqrt{2 k_{\beta} L_{\beta} \sin ^{2}\left(\frac{\theta_{\beta}+\theta}{2}\right)}
\end{aligned}
$$

are the detour parameters. ${ }^{8}$

${ }^{1} \mathrm{P}$. Ya Ufimtsev, Fundamentals of the Physical Theory of Diffraction (Wiley, NJ, 2007), Chap. 3, pp. 59-68; Chap. 4, pp. 71-76.

${ }^{2}$ B. Lü, M. Darmon, L. Fradkin, and C. Potel, "Numerical comparison of acoustic wedge models, with application to ultrasonic telemetry," Ultrasonics, in press (2015).

${ }^{3}$ V. Zernov, L. Fradkin, and M. Darmon, "A refinement of the Kirchhoff approximation to the scattered elastic fields," Ultrasonics 52, 830-835 (2012).

${ }^{4}$ J. B. Keller, "Geometrical theory of diffraction," J. Opt. Soc. Am. 52, 116-130 (1962).

${ }^{5}$ J. D. Achenbach and A. K. Gautesen, "Geometrical theory of diffraction for three-D elastodynamics," J. Acoust. Soc. Am. 61, 413-421 (1977).

${ }^{6}$ J. D. Achenbach, A. K. Gautesen, and H. McMaken, Rays Methods for Waves in Elastic Solids (Pitman, New York, 1982), Chap. 5, pp. $109-148$.

${ }^{7}$ R. M. Lewis and J. Boersma, "Uniform asymptotic theory of edge diffraction," J. Math. Phys. 10, 2291-2305 (1969).

${ }^{8}$ S. W. Lee and G. A. Deschamps, "A uniform asymptotic theory of electromagnetic diffraction by a curved wedge," IEEE Trans. Antennas. Propag. 24, 25-34 (1976).

${ }^{9}$ D. S. Ahluwalia, "Uniform asymptotic theory of diffraction by the edge of a three dimensional body," SIAM J. Appl. Math. 18, 287-301 (1970).

${ }^{10}$ B. L. Van Der Waerden, "On the method of saddle points," Appl. Sci. Res., Sect. B 2, 33-45 (1951). 
${ }^{11}$ P. H. Pathak and R. G. Kouyoumjian, "The dyadic diffraction coefficient for a perfectly-conducting wedge,” DTIC Document, Tech. Rep. (1970).

${ }^{12}$ R. G. Kouyoumjian and P. H. Pathak, "A uniform geometrical theory of diffraction for an edge in a perfectly conducting surface," Proc. IEEE 62 , 1448-1461 (1974).

${ }^{13} \mathrm{P}$. C. Clemmow, "Some extension to the method of integration by steepest descent," Q. J. Mech., Appl. Math. III, 241-256 (1950).

${ }^{14}$ V. A. Borovikov and B. Y. Kinber, Geometrical Theory of Diffraction (Institution of Electrical Engineers, London 1994), Sec. 3.7.

${ }^{15} \mathrm{H}$. McMaken, "A uniform theory of diffraction for elastic solids," J. Acoust. Soc. Am. 75, 1352-1359 (1984).

${ }^{16} \mathrm{~N}$. Tsingos, T. Funkhouser, A. Ngan, and I. Carlbom, "Modeling acoustics in virtual environments using the uniform theory of diffraction," Proc. ACM SIGGRAPH (2001), pp. 545-552.

${ }^{17}$ M. F. Catedra, J. Perez, F. Saez de Adana, and O. Gutierrez, "Efficient ray-tracing techniques for three-dimensional analyses of propagation in mobile communications: Application to picocell and microcell scenarios," IEEE Antennas Propag. Mag. 40, 15-28 (1998).

${ }^{18} \mathrm{D}$. Bouche and F. Molinet, Méthodes Asymptotiques en Électromagnétisme (Asymptotic Methods in Electromagnetics) (SpringerVerlag, Berlin Heidelberg, 1994), Chap. 5, pp. 192-198.

${ }^{19}$ D. Bouche, F. Molinet, and R. Mittra, Asymptotic Methods in Electromagnetics (Springer-Verlag, Berlin Heidelberg, 1997), Chap. 5.

${ }^{20} \mathrm{~V}$. A. Borovikov, Uniform Stationary Phase Method (The Institution of Electrical Engineers, London, 1994), pp. 159-161.

${ }^{21}$ F. Molinet, Acoustic High-Frequency Diffraction Theory (Momentum Press, New York, 2011), Chap. 3, pp. 244-259.
${ }^{22}$ L. Ju. Fradkin and R. Stacey, "The high-frequency description of scatter of a plane compressional wave by an elliptical crack," Ultrasonics 50, 529-538 (2010).

${ }^{23} \mathrm{D}$. Gridin, "High-frequency asymptotic description of head waves and boundary layers surrounding critical rays in an elastic half-space," J. Acoust. Soc. Am. 104, 1188-1197 (1998).

${ }^{24}$ J. D. Achenbach and A. K. Gautesen, "Edge diffraction in acoustics and elastodynamics," in Low and High Frequency Asymptotics 2 (Elsevier, New York, 1986), Chap. 4, pp. 335-401.

${ }^{25}$ L. W. Schmerr and S.-J. Song, Ultrasonic Nondestructive Evaluation Systems: Models and Measurements (Springer, New York, 2007).

${ }^{26} \mathrm{M}$. Darmon and S. Chatillon, "Main features of a complete ultrasonic measurement model - Formal aspects of modeling of both transducers radiation and ultrasonic flaws responses," Open J. Acoust. 3A, 43-53 (2013).

${ }^{27}$ M. Darmon, V. Dorval, A. Kamta Djakou, L. Fradkin, and S. Chatillon, "A system model for ultrasonic NDT based on the physical theory of diffraction (PTD)," Ultrasonics 64, 115-127 (2016).

${ }^{28} \mathrm{G}$. Toullelan, R. Raillon, S. Chatillon, V. Dorval, M. Darmon, and S. Lonné, "Results of the 2015 UT modeling benchmark obtained with models implemented in CIVA," AIP Conf. Proc. in press (2016).

${ }^{29}$ A. Kamta Djakou, M. Darmon, and C. Potel, "Elastodynamic models for extending GTD to penumbra and finite size scatterers," Phys. Proc. 70, 545-549 (2015)

${ }^{30}$ M. Darmon, N. Leymarie, S. Chatillon, and S. Mahaut, "Modelling of scattering of ultrasounds by flaws for NDT," in Ultrasonic Wave Propagation in Non Homogeneous Media (Springer, Berlin, 2009), Vol. 128, pp. 61-71. 